\title{
Telehealth Delivery of Tobacco Cessation Treatment in Cancer Care: An Ongoing Innovation Accelerated by the COVID-19 Pandemic
}

\author{
The Cancer Center Cessation Initiative Telehealth Working Group*
}

\begin{abstract}
The COVID-19 pandemic precipitated a rapid transformation in healthcare delivery. Ambulatory care abruptly shifted from in-person to telehealth visits with providers using digital video and audio tools to reach patients at home. Advantages to telehealth care include enhanced patient convenience and provider efficiencies, but financial, geographic, privacy, and access barriers to telehealth also exist. These are disproportionately greater for older adults and for those in rural areas, low-income communities, and communities of color, threatening to worsen preexisting disparities in tobacco use and health. Pandemic-associated regulatory changes regarding privacy and billing allowed many Cancer Center Cessation Initiative (C3I) programs in $\mathrm{NCl}$-designated Cancer Centers to start or expand video-based telehealth care. Using $3 \mathrm{C} 3 \mathrm{l}$ programs as examples, we describe the methods used to shift to telehealth delivery. Although telephone-delivered treatment was already a core tobacco treatment modality with a robust evidence base, little research has yet compared the effectiveness of tobacco cessation treatment delivery by video versus phone or in-person modalities. Video-delivery has shown greater medication adherence, higher patient satisfaction, and better retention in care than phone-based delivery, and may improve cessation outcomes. We outline key questions for further investigation to advance telehealth for tobacco cessation treatment in cancer care.

J Natl Compr Canc Netw 2021;19(Suppl 1):S21-24 doi: $10.6004 /$ jnccn.2021.7092
\end{abstract}

\section{Background}

The COVID-19 pandemic precipitated a rapid transformation in US healthcare delivery. Providers and systems abruptly shifted from in-person to virtual telehealth visits using digital video and audio tools. ${ }^{1}$ Healthcare systems rapidly re-engineered health information technology and workflows, facilitated by changes in payment regulations that permitted billing for remotely delivered services not previously allowed. ${ }^{1}$ The success of this shift demonstrated the viability of remote care delivery. Healthcare systems' current challenge is defining how best to integrate and sustain telehealth care for the postpandemic era.

Delivering care to patients using video- or phonebased telehealth tools offers many potential benefits. Treatment delivered at home increases access to care for many patients, allows family members' participation in care, and may give providers additional information about patients' needs. Telehealth care may increase the efficiency and ease of delivering services that do not require an in-person visit, thereby increasing patient

*A complete list of the collaborators in the Cancer Center Cessation Initiative Telehealth Working Group appears at the end of this article. and provider satisfaction with care. However, telehealth care also has challenges, including financial, geographic, and access-related barriers. ${ }^{1}$ Patient barriers are disproportionately greater for older adults and for those in low-income communities, rural areas, and communities of color, threatening to worsen preexisting disparities in healthcare access and health status. ${ }^{2,3}$ Although technology barriers could worsen preexisting disparities in some cases, there are opportunities in which technology may substantially increase reach and communication in cohorts with limited financial support and difficulty with travel. Although HIPAA-compliant digital platforms support privacy and confidentiality, privacy barriers to virtual care delivery have not been fully or permanently resolved. And finally, although some states have mandated third-party reimbursement telehealth commensurate with in-person visits, video and voice telehealth care delivery still operate under temporary Medicare rules. ${ }^{4}$

\section{Telehealth and Tobacco Use Treatment Delivery} The shift to telehealth was arguably less dramatic for tobacco use treatment services, because telephone visits are a longstanding, core treatment modality in 
community and healthcare settings. ${ }^{5,6}$ Quitlines, the largest tobacco treatment network in the United States, already offered tobacco users behavioral treatment by telephone and web-based interfaces plus short-term nicotine replacement. These services required only a telephone and were generally provided free of charge. State-based telephone quitlines have been used to improve access to support for quitting smoking in patients with cancer. ${ }^{7}$ However, quitlines typically provide brief interventions and focus on tobacco users who are ready to quit. ${ }^{8}$

Because patients with cancer experience unique stressors that can inhibit cessation, ${ }^{9}$ the NCCN Clinical Practice Guidelines in Oncology (NCCN Guidelines) for treating tobacco use among patients with cancer recommend a more robust treatment than currently provided by quitlines or many community programs. ${ }^{10}$ Prepandemic, the Cancer Center Cessation Initiative (C3I) had enabled many NCI-designated Cancer Centers to provide in-person and/or remotely delivered cessation services. When the pandemic required suspension of inperson visits, most C3I cancer centers shifted their service delivery from in-person to remote visits using telephone and/or video telehealth services. A survey of 41 C3I sites reported an increase in the proportion of sites offering phone-based counseling from $71 \%$ to $84 \%$ between December 2019 and December 2020, with most indicating that this shift was in response to COVID-19.

\section{Case Studies of Telehealth Adoption}

We describe 3 C3I programs that transitioned from primarily in-person to virtual telehealth services prompted by the COVID-19 pandemic (Table 1).
Memorial Sloan Kettering Cancer Center (MSKCC) and The University of Text MD Anderson Cancer Center (MDACC) have well-established comprehensive tobacco treatment programs, introduced in 1999 (MSKCC) and 2006 (MDACC). Prepandemic, both programs offered in-person individual and/or group visits, and team members prescribed cessation medications. Both were already piloting telehealth service delivery pre-pandemic but efforts were limited by state medical board restrictions on prescribing medications by phone or providing counseling across state lines. When COVID constrained in-person service delivery, these regulatory barriers were lifted, and both programs rapidly shifted to remote telehealth service delivery in March 2020. For both programs, the switch to telehealth increased patients' access to comprehensive tobacco treatment services. In particular, the proportion of patients completing scheduled visits increased substantially after the switch to telehealth (MSKCC, 60\%-75\% ${ }^{11}$; MDACC, $79 \%-91 \%)$. Because interpreters could easily join telehealth visits, MSKCC reached more diverse and non-English-speaking populations that previously had difficulty accessing in-person services, and also reached more patients living far from the facility. However, providers and patients at both programs also faced technical challenges. At MDACC, many patients were reluctant to schedule video-based visits, despite the program's offer to provide technical support. Consequently, $80 \%$ to $90 \%$ of services were delivered only by telephone during the pandemic's first months. By early 2021, however, $50 \%$ of visits were provided by video telehealth, which the program attributed to increasing public familiarity with video conferencing.

Table 1. Examples of Telehealth Adaptation of Tobacco Treatment That Occurred During the Pandemic

\begin{tabular}{|c|c|c|c|}
\hline & $\begin{array}{l}\text { Memorial Sloan Kettering } \\
\text { Cancer Center (MSKCC) } \\
\text { (New York \& New Jersey) }\end{array}$ & $\begin{array}{l}\text { MD Anderson Cancer } \\
\text { Center (MDACC) } \\
\text { (Houston, Texas) }\end{array}$ & $\begin{array}{l}\text { University of } \\
\text { Chicago Medicine (UCM) } \\
\text { (Chicago, Illinois) }\end{array}$ \\
\hline $\begin{array}{l}\text { Treatment Modalities } \\
\text { Pre-COVID-19 }\end{array}$ & $\begin{array}{l}\text { Primarily phone visits, with in- } \\
\text { person individual and group visits } \\
\text { and limited use of video telehealth }\end{array}$ & $\begin{array}{l}\text { Primarily in-person individual visits } \\
\text { Piloting video telehealth to patient } \\
\text { homes }\end{array}$ & Primarily in-person group visits \\
\hline $\begin{array}{l}\text { Treatment Modalities } \\
\text { During COVID-19 }\end{array}$ & $\begin{array}{l}\text { Exclusive telehealth or phone. } \\
\text { Platforms - } 3 / 20 \text { : Cisco Jabber app } \\
\text { linked to patient portal; Doximity } \\
\text { or Face-time as backup. } 4 / 21 \text { : } \\
\text { transition to Zoom for group } \\
\text { telehealth }\end{array}$ & $\begin{array}{l}\text { Primarily telehealth Zoom } \\
\text { platform, with phone call backup }\end{array}$ & $\begin{array}{l}\text { Exclusive telehealth Zoom } \\
\text { platform }\end{array}$ \\
\hline Service Delivery Staff ${ }^{a}$ & Psychologist/TTS, APN/TTS & $\begin{array}{l}\text { MD, PA-C, LPC, LPCA, LCSW, } \\
\text { Psychologist }\end{array}$ & Psychologist, LCSW/TTS \\
\hline Bill for Services? ${ }^{a}$ & Yes & No & Yes \\
\hline Patients Served ${ }^{\mathrm{a}}$ & $\begin{array}{l}\text { Cancer center patients, some } \\
\text { household members }\end{array}$ & $\begin{array}{l}\text { Cancer center patients and } \\
\text { household members }\end{array}$ & $\begin{array}{l}\text { Cancer center patients and } \\
\text { patients with other medical } \\
\text { conditions }\end{array}$ \\
\hline
\end{tabular}

Abbreviations: APN, Advanced Practice Nurse (eg, Nurse Practitioner); LCSW, Licensed Clinical Social Worker; LPC, Licensed Professional Counselor; LPCA, Licensed Professional Counseling Associate; PA-C, Physician Assistant; TTS, Tobacco Treatment Specialist.

${ }^{a}$ Before and during COVID-19 pandemic. 
University of Chicago Medicine (UCM) has a newer tobacco treatment program, established in 2016, offering in-person group programs with behavioral support, free nicotine replacement therapy, and medication prescriptions from primary care providers. To enhance program reach, referrals from clinicians were supplemented with proactive outreach to Cancer Center patients using a system of automated phone calls and emails that connected interested patients to medication and counseling resources. During the pandemic, in-person groups transitioned to a virtual format using a HIPAA-compliant Zoom platform. The attendance rate of scheduled groups increased from $47 \%$ (onsite in 2019) to $57 \%$ (virtual in 2020). As with most programs, the volume of scheduled sessions in 2020 was consistent with pre-pandemic levels after an initial slowdown of outpatient services and referrals. Many patients reported that the virtual format reduced barriers such as public transportation, parking charges, and mobility issues, but other patients remained reluctant or unable to connect via video. These barriers were particularly relevant for UCM's predominantly low-income, Black/African American patient population.

\section{Research Agenda and Questions for the Future}

The telehealth transformation of tobacco treatment delivery during the pandemic may offer the opportunity to better tailor treatment delivery to the needs of patients with cancer who use tobacco. Despite robust evidence demonstrating the effectiveness of delivering telephonebased tobacco treatment, ${ }^{5}$ limited research has compared delivering treatment by video vs phone vs other modalities. A recent Cochrane review found no difference in cessation outcomes between video- and phone-based counseling in the general population, but also noted limitations across studies and the need for additional trials. ${ }^{5}$ Importantly, compared with phone-based delivery, videodelivery has shown greater medication adherence and higher patient satisfaction. ${ }^{12,13}$ Finally, initial 4-month cessation outcomes from an ongoing randomized controlled trial in rural Australia, comparing video versus phone versus mailed materials, indicate that video was significantly more effective than mailed materials, but did not differ from phone-based counseling. ${ }^{14}$

To advance the field of tobacco-specific telehealth research in cancer care, we suggest several important areas for further investigation.

Operations: Feasibility and utilization of video-based and phone-based telehealth:

- Identify the training needs for staff to transition to virtual care delivery

- Identify and investigate patient barriers

- Do patients prefer telephone versus video visits?
- Does providing technical assistance prior to intake facilitate successful engagement?

- How can we best facilitate patients' willingness to accept video visits?

- What is the feasibility and uptake of group versus individual video visits?

- Are there any additional/unique barriers by race/ ethnicity and socioeconomic status?

- How do we reduce internet access barriers to accommodate video visits?

- Assess the feasibility and timing (relative to diagnosis/treatment) of including tobacco treatment specialists during virtual oncology telehealth visits, given the potential to facilitate "warm handoffs" from oncology provider

- Assess the feasibility of continued delivery across state lines

- Identify billing issues (eg, low reimbursement rates of all tobacco use treatment services)

- Assess the costs and benefits of integrating remote assessments (eg, carbon monoxide monitors for at-home use)

Outcomes: Reach and effectiveness of video-based telehealth compared with in-person or telephone:

- Reach of patients offered tobacco treatment, percentage who accept each treatment modality

- Regarding the "digital divide": describe the patient characteristics of those who do/do not use virtual (telephone or video) care

- To what extent does telehealth assist rural and urban populations with transportation challenges?

- Does telehealth access increase pandemic-related readiness to quit?

- Patient engagement: visit completion, patient satisfaction scores, therapeutic alliance

- Patient receipt of tobacco treatment: number of completed sessions, percentage who complete the full program, use/duration of pharmacological treatment

- Treatment effectiveness: quit attempts, abstinence at end of treatment and longer term (6-12 months), stratified by demographic, oncology treatment regimen, and clinical characteristics

- What is the optimal combination of virtual and inperson care?

- Provider efficiency (number of patients treated per day), patient satisfaction, quality of treatment delivery

- Comparison of treatment modalities on cost, revenue, cost-effectiveness, and sustainability

- Importance of payors maintaining reimbursement for phone-based telehealth until connectivity issues for video-based telehealth have been resolved 


\section{Conclusions}

Advancing telephone and video-based telehealth has the potential to improve tobacco cessation treatment in cancer care settings and beyond. It may be particularly salient for marginalized populations. The COVID-19 pandemic catalyzed telehealth scale-up, offering the opportunity to investigate whether this treatment delivery modality can improve outcomes in tobacco cessation and cancer care.

\section{Cancer Center Cessation Initiative Telehealth Working Group Members}

Nancy A. Rigotti, MD ${ }^{1, *}$; Kathryn L. Taylor, $\mathrm{PhD}^{2, *}$; Diane Beneventi, PhD ${ }^{3,+}$; Andrea King, PhD ${ }^{4,+}$; Chris Kotsen, PsyD, NCTTP ${ }^{5,+}$; Linda Fleisher, PhD, $\mathrm{MPH}^{6,+}$; Adam O. Goldstein, MD, MPH ${ }^{7,+}$; Elyse R. Park, PhD, MPH ${ }^{8,+}$; Scott E. Sherman, MD, MPH ${ }^{9, \dagger}$; Michael B. Steinberg, MD, MPH ${ }^{10,+}$; David A. Albert, DDS, MPH ${ }^{11, \pm} ;$ Lisa Sanderson Cox, PhD ${ }^{12, \pm}$; Rashelle B. Hayes, PhD ${ }^{13, \pm}$; Sarah D. Hohl, PhD, MPH ${ }^{14, \pm}$; Christine E. Sheffer, PhD 15, ; Kimberly A. Shoenbill, MD, PhD ${ }^{16, \pm}$; Vani N. Simmons, PhD 17, ; Graham W. Warren, MD, $\mathrm{PhD}^{18, *}$; Robert Adsit, MEd ${ }^{19, \$}$; Mara Minion, MA ${ }^{14, \$} ;$ Danielle Pauk, BS ${ }^{14, \$} ;$ and Betsy Rolland, PhD, MLIS, MPH ${ }^{14,20, \S}$

${ }^{1}$ Tobacco Research and Treatment Center, Mongan Institute, Department of Medicine, Massachusetts General Hospital, Harvard Medical School, Boston, Massachusetts; ${ }^{2}$ Cancer Prevention and Control Program, Lombardi Comprehensive Cancer Center, Georgetown University Medical Center, Washington, DC; ${ }^{3}$ Department of Behavioral Science, University of Texas MD Anderson Cancer Center, Houston, Texas; ${ }^{4}$ Department of Psychiatry and Behavioral Neuroscience, University of Chicago, Chicago, Illinois;

${ }^{5}$ Department of Psychiatry and Behavioral Sciences, Memorial Sloan Kettering Cancer Center, New York, New York; ${ }^{6}$ Cancer Prevention and Control, Fox Chase Cancer Center, Philadelphia, Pennsylvania; ${ }^{7}$ Department of Family Medicine and Lineberger Comprehensive Cancer Center, UNC School of Medicine, Chapel Hill, North Carolina; ${ }^{8}$ Department of Psychiatry,

Massachusetts General Hospital, Boston, Massachusetts; ${ }^{9}$ Department of
Population Health, New York University School of Medicine, New York, New York; ${ }^{10}$ Department of Medicine, Rutgers Robert Wood Johnson Medical School; Cancer Institute of New Jersey; and Rutgers Center for Tobacco Studies, New Brunswick, New Jersey; ${ }^{11}$ Department of Dentistry and Public Health, Columbia University Irving Medical Center, New York, New York; ${ }^{12}$ Department of Population Health, University of Kansas Medical Center, Kansas City, Kansas; ${ }^{13}$ Massey Cancer Center, Virginia Commonwealth University, Richmond, Virginia; ${ }^{14}$ Carbone Cancer Center, School of Medicine and Public Health, University of Wisconsin-Madison, Madison, Wisconsin; ${ }^{15}$ Department of Health Behavior, Roswell Park Comprehensive Cancer Center, Buffalo, New York; ${ }^{16}$ Department of Family Medicine, University of North Carolina, Chapel Hill, North Carolina; ${ }^{17}$ Department of Health Outcomes and Behavior, Moffitt Cancer Center, Tampa, Florida;

${ }^{18}$ Department of Radiation Oncology, and Department of Cell and Molecular Pharmacology and Experimental Therapeutics, Hollings Cancer Center, Medical University of South Carolina, Charleston, South Carolina;

${ }^{19}$ Department of Medicine, Center for Tobacco Research and Intervention, School of Medicine and Public Health, University of Wisconsin-Madison, Madison, Wisconsin; and ${ }^{20}$ Institute for Clinical and Translational Research, University of Wisconsin-Madison, Madison, Wisconsin.

"Working Group Chair; 'Writing Group Author; ${ }^{\text {}}$ Working Group Author: ${ }^{\S}$ Coordinating Center Author.

Submitted July 26, 2021; final revision received September 14, 2021; accepted for publication September 16, 2021.

Disclosures: C. Kotsen has disclosed serving on an advisory board for the LuCa National Training Network at the University of Louisville. G.W. Warren has disclosed participating in research for the Agency for Healthcare Research and Quality and serving as a consultant for the Canadian Partnership Against Cancer. The remaining individuals have disclosed no relevant financial relationships.

Funding: This supplement was funded by the $\mathrm{C} 3 \mathrm{I}$ Coordinating Center contract from the National Cancer Institute (CRDF Award \#66590). In addition, authors received funding for their $\mathrm{C} 3 \mathrm{I}$ participation via a supplement to their NCI P30 cancer center support grant during the period 2017-2021.

Correspondence: Nancy A. Rigotti, MD, Division of General Internal Medicine, Massachusetts General Hospital, 100 Cambridge Street, Suite 1600, Boston, MA 02114. Email: Rigotti.Nancy@mgh.harvard.edu

\section{References}

1. Clancy $C M$, Kirsh S. Virtual care and the pandemic: are we reaching all patients? Ann Intern Med 2021;174:116-117.

2. Hsiao V, Chandereng T, Lankton RL, et al. Disparities in telemedicine access: a cross-sectional study of a newly established infrastructure during the COVID-19 pandemic. Appl Clin Inform 2021;12:445-458.

3. Cantor JH, McBain RK, Pera MF, et al. Who is (and is not) receiving telemedicine care during the COVID-19 pandemic. Am J Prev Med 2021;61: 434-438.

4. American Academy of Family Physicians. Tobacco cessation telehealth guide. Accessed June 18, 2021. Available at: https://www.aafp.org/dam/ AAFP/documents/patient_care/tobacco/tobacco-cessation-telehealthguide.pdf

5. Matkin W, Ordóñez-Mena JM, Hartmann-Boyce J. Telephone counselling for smoking cessation. Cochrane Database Syst Rev 2019;5:CD002850.

6. US Department of Health and Human Services. Smoking cessation: a report of the Surgeon General. Accessed September 6, 2021. Available at: https://www.hhs.gov/sites/default/files/2020-cessation-sgr-full-report.pdf

7. Notier AE, Hager P, Brown KS, et al. Using a quitline to deliver opt-out smoking cessation for cancer patients. JCO Oncol Pract 2020;16:e549-556.

8. North American Quitline Association. Results from the 2020 NAQC Annual Survey of Quitlines. Accessed June 19, 2021. Available at: https://www.naquitline.org/page/2020survey
9. Park ER, Perez GK, Regan S, et al. Effect of sustained smoking cessation counseling and provision of medication vs shorter-term counseling and medication advice on smoking abstinence in patients recently diagnosed with cancer: a randomized clinical trial. JAMA 2020;324:1406-1418.

10. Shields PG, Herbst RS, Arenberg D, et al. Smoking Cessation, Version 1.2016, NCCN Clinical Practice Guidelines in Oncology. J Natl Compr Canc Netw 2016;14:1430-1468.

11. Kotsen C, Dilip D, Carter-Harris $L$, et al. Rapid scaling up of telehealth treatment for tobacco-dependent cancer patients during the COVID-19 outbreak in New York City. Telemed J E Health 2021;27:20-29.

12. Richter KP, Shireman TI, Ellerbeck EF, et al. Comparative and cost effectiveness of telemedicine versus telephone counseling for smoking cessation. J Med Internet Res 2015;17:e113.

13. Byaruhanga J, Wiggers $\mathrm{J}$, Paul $\mathrm{CL}$, et al. Acceptability of real-time video counselling compared to other behavioural interventions for smoking cessation in rural and remote areas. Drug Alcohol Depend 2020;217: 108296.

14. Byaruhanga J, Paul CL, Wiggers J, et al. The short-term effectiveness of real-time video counselling on smoking cessation among residents in rural and remote areas: an interim analysis of a randomised trial. J Subst Abuse Treat 2021;131:108448 